\title{
Qualidade do valor da medida de massa corporal nos Centros Municipais de Saúde do Município do Rio de Janeiro, 1996
}

\author{
Quality of body mass measurement \\ in Rio de Janeiro primary health care \\ facilities in 1996
}

Jane de Carlos Santana Capelli 1

Luiz Antonio dos Anjos 2,3

Inês Rugani Ribeiro de Castro 4,5

\footnotetext{
1 Departamento de

Enfermagem e Departamento

de Educação Física,

Universidade Iguaçu.

Av. Abílio Augusto Távora

2134, Nova Iguaçu, RJ

26260-000, Brasil.

2 Laboratório de Avaliação

Nutricional e Funcional,

Departamento de Nutrição

Social, Universidade

Federal Fluminense.

Rua São Paulo 30

Niterói, $R J$

24040-115, Brasil.

3 Centro de Estudos da Saúde

do Trabalhador e Ecologia

Humana, Escola Nacional

de Saúde Pública,

Fundação Oswaldo Cruz.

Rua Leopoldo Bulhões 1480,

Rio de Janeiro, $R J$

21041-210, Brasil.

${ }^{4}$ Instituto de Nutrição

Annes Dias. Av. Pasteur 44,

Rio de Janeiro, $R J$

22290-240, Brasil.

5 Departamento de Nutrição Social, Instituto de Nutrição,

Universidade do Estado

do Rio de Janeiro.

Rua São Francisco Xavier

524, sala 12019, 12o andar,

Bloco D, Rio de Janeiro, RJ

20550-013, Brasil.
}

\begin{abstract}
The main purpose of the study was to evaluate the quality of body mass measurement of children under 5 years of age treated in 21 health care units in the city of Rio de Janeiro. A total of 38 health care professionals were observed, 292 children were weighed, and 41 scales were calibrated. Almost all of the mechanical scales were positioned improperly. The procedure of weighing the child "undressed" was not performed in 29.9\% of the cases on adult beam scales. Almost all of the scales were calibrated (98\%) and showed a high correlation coefficient (0.999). There were no important differences between body mass values obtained by the health care professionals and those read by the observer. Thus, nutritional classification using either value showed a perfect correlation (Kappa correlation coefficient $=1$ ). Although some procedures in obtaining body mass values were unsatisfactory, they did not affect the nutritional classification of children in the present study.
\end{abstract}

Key words Anthropometry; Infant Nutrition; Equipment

Resumo O estudo objetivou avaliar a qualidade do valor da medida de massa corporal de crianças menores de cinco anos atendidas nos Centros Municipais de Saúde (CMS) do Município do Rio de Janeiro. Observaram-se 38 profissionais de saúde realizando as atividades de medição da massa corporal de 292 crianças atendidas num turno de atendimento de cada um dos 21 CMS, sendo calibrados 41 equipamentos de pesagem. As balanças mecânicas (pediátricas ou de plataforma), na sua grande maioria, estavam inadequadamente posicionadas. Os procedimentos básicos de medição em equipamentos mecânicos não foram realizados de forma correta na maior parte das vezes. O procedimento de "despir a criança" não foi realizado em 29,9\% nas balanças de plataforma e, dos realizados, 42,3\% o foram incorretamente. Houve pequenas diferenças entre os valores de massa corporal produzidos pelos profissionais de saúde e os lidos pelo observador, não alterando a classificação nutricional que mostrou concordância perfeita (coeficiente de Kappa =1). Apesar de alguns resultados não estarem satisfatórios para a produção do valor da medida de massa corporal na rotina dos serviços, os valores produzidos pelos profissionais de saúde não comprometeram as classificações nutricionais das crianças avaliadas neste estudo.

Palavras-chave Antropometria; Nutrição Infantil; Equipamento 


\section{Introdução}

O corpo humano é constituído de ossos, gordura, músculos, glândulas, vísceras e fluidos que compõem a sua massa corporal total. $\mathrm{O}$ valor da medida de massa corporal é, na prática clínica, mais conhecido como peso e sua unidade de base é o quilograma (IEL, 1994). Desde as primeiras etapas do crescimento do ser humano, a massa corporal sofre variações fisiológicas, como flutuações na composição corporal causadas pelos níveis de ingestão alimentar e de hidratação (Mueller \& Martorell, 1988). Entretanto, estas variações se tornam um motivo de preocupação quando ocorrem alterações patológicas tanto por problemas nutricionais (déficits ou ganhos excessivos) como por agressões infecciosas, metabólicas e psicológicas.

Na área da saúde, uma forma de acompanhar estas variações é a utilização do valor da medida de massa corporal que, quando combinado ao de outras medidas, como a idade e a estatura, torna-se um importante instrumento de avaliação nutricional (Batista Filho, 1994), especialmente nos primeiros anos de vida, permitindo acompanhar os diversos estágios do crescimento (WHO, 1995), podendo, ainda, indicar a qualidade de vida e o nível de desenvolvimento de uma população (Mason et al., 1984; Monteiro et al., 1992).

O valor da medida de massa corporal é amplamente utilizado na avaliação antropométrica por ser de fácil obtenção quando comparado a outras medidas. Contudo, é passível de tendenciosidades e erros de mensuração. De forma geral, os erros existentes na tomada do valor da medida de massa corporal em unidades de saúde podem ocorrer pela falta de: treinamento de pessoal quanto à técnica de pesagem (podendo ocorrer erros de leitura e registro de dados), bem como reciclagem periódica dos mesmos; rotatividade de profissionais na coleta da medida, pois, com o tempo, pode haver erros de mensuração devido ao cansaço, desatenção e/ou sobrecarga de atividades; utilização adequada dos instrumentos; verificação do estado de conservação dos equipamentos de pesagem; manutenção e calibração periódicas dos mesmos; disposição adequada dos equipamentos no local de pesagem; apoio adequado para os equipamentos de pesagem; ambiente de trabalho favorável com sala ampla para permitir liberdade de movimento, piso plano, temperatura ambiental normal, sem correntes de ar (INAN/MS, 1996; INMETRO, 1995).

A tomada da medida de massa corporal deve ser realizada por pessoas previamente capacitadas na perspectiva de se evitar erros ou problemas decorrentes do processo de mensuração (Habicht, 1974) e, conseqüentemente, garantir um valor de qualidade. Como já foi descrito anteriormente, esta condição isoladamente não é suficiente para se evitar erro no valor da medida. Isto porque existem outros fatores também relevantes que, despercebidos, influenciam o valor obtido, tornando-o duvidoso quanto a sua exatidão, podendo, posteriormente, comprometer possíveis diagnósticos individuais e/ou coletivos, além de comprometer os procedimentos que dependem da medida, como os cálculos da dosagem de medicamentos (Dornbrand et al., 1990), do valor de massa corporal teórico e das necessidades energéticas (Chaves, 1978).

O presente estudo objetivou avaliar a qualidade do valor da medida de massa corporal, para assim retratar a realidade que envolve o seu processo de produção na rotina dos serviços de saúde, já que os profissionais de saúde necessitam de valores fidedignos desta medida para desenvolver as atividades de assistência que lhes cabem.

\section{Materiais e métodos}

\section{População e área de estudo}

Para avaliar a qualidade dos valores das medidas de massa corporal de crianças menores de cinco anos foram estudados todos os 21 Centros Municipais de Saúde (CMS) do Município do Rio de Janeiro, sendo cada um visitado em um turno de atendimento escolhido aleatoriamente por sorteio. Ao todo foram observados 38 profissionais de saúde realizando as atividades de mensuração da massa corporal de 292 crianças menores de cinco anos em 41 equipamentos nos 21 CMS visitados.

\section{Métodos}

A coleta de dados foi feita por um observador e um assistente previamente treinados, que eram encaminhados à sala de medição antropométrica e iniciavam suas atividades anotando informações gerais sobre a unidade e a sala em um questionário desenvolvido para o estudo. As informações sobre a implantação do Sistema de Vigilância Alimentar e Nutricional (SISVAN) eram colhidas com o diretor do CMS ou com o coordenador do programa de atendimento integral à saúde após as atividades de calibração. Isto porque uma das etapas do processo de implantação do SISVAN é o treinamento dos profissionais quanto à técnica de 
pesagem. Assim, dependendo do tempo da sua implantação na unidade de saúde, haveria a possibilidade de se avaliar o tempo em que ocorreu o treinamento dos mesmos.

A pesquisadora (observador) se apresentava ao profissional de saúde responsável pela medição da massa corporal como estagiária que iria observar as medições. Quando as atividades de pesagem eram iniciadas, o observador averiguava, seguindo roteiro padronizado, se os procedimentos de medição da massa corporal estavam sendo realizados e, se sim, se estavam corretos ou não. Os procedimentos consistiam em: nivelar o fiel da balança; despir a criança; colocar a criança no centro da balança; esperar a criança ficar quieta; mover o cursor dos quilos; mover o cursor dos gramas; esperar a agulha do braço da balança ficar alinhada com o fiel; ler o valor de massa corporal de frente aos marcadores da balança; registrar o valor de massa corporal.

Finalizada a medição da criança pelo enfermeiro ou auxiliar de enfermagem, o observador lia o valor da medida de massa corporal na escala da balança e o anotava no roteiro padronizado ao lado do valor produzido pelo profissional de saúde e que havia sido anotado no cartão da criança ou prontuário. A data de nascimento também foi copiada dessas fontes. Em 42 crianças não foi possível observar o valor. Assim, as comparações entre o profissional de saúde e o observador foram realizadas para os valores de 250 crianças. Ao final do turno de observação, era realizado o processo de calibração das balanças pediátricas (mecânicas ou eletrônicas) e de plataforma (mecânicas).

$O$ roteiro de técnica de pesagem foi adaptado com base em várias publicações (INAN/MS, s.d., 1996; Naciones Unidas, 1988). A planilha de calibração foi preparada com a ajuda de profissionais do Laboratório de Massas (LAMAS) do Instituto Nacional de Metrologia, Normalização e Qualidade Industrial (INMETRO). Todos os instrumentos foram previamente testados e validados em estudo piloto.

Quanto ao estado de conservação dos equipamentos, foram contemplados os seguintes aspectos para que o equipamento fosse recomendado à manutenção: dificuldades em se manusear o calibrador, pontos de ferrugem no prato ou no braço da balança, calços (pedaço de papelão ou madeira) na base da balança.

\section{Análise dos dados}

Os resultados foram descritos quantitativamente por intermédio de tabelas de freqüência relativa e absoluta e analisados numa aborda- gem quanti-qualitativa, utilizando-se, além da literatura, as observações realizadas, pelo observador, da rotina da sala de preparo.

Foi analisada a concordância entre os valores das medidas de massa corporal calculandose a diferença (DV) entre o valor produzido pelos profissionais de saúde (V) e o lido pelo observador $\left(V_{1}\right)$, ou seja, $D V=V-V_{1}$. Para saber se esta variação era aceitável, foram propostos intervalos de adequação para cada tipo de balança (pediátrica - mecânica e eletrônica - e plataforma), a partir da resolução dos mesmos. Os intervalos estabelecidos foram: balança pediátrica eletrônica $=-5 g$ a $+5 g$; balança pediátrica mecânica $=-10 \mathrm{~g}$ a $+10 \mathrm{~g}$; balança de plataforma mecânica $=-100 \mathrm{~g} \mathrm{a}+100 \mathrm{~g}$.

Classificou-se o estado nutricional de cada criança (baixo peso, adequado e sobrepeso) utilizando-se o índice massa corporal para idade (MC/I) com os pontos de corte de -2 e +2 escores z da curva de referência NCHS (1977) para baixo peso e sobrepeso, respectivamente. Para avaliar a concordância entre as classificações nutricionais, foram utilizados os valores das medidas de massa corporal obtidos pelo profissional e pelo observador com correções para a calibração da balança e o fato da vestimenta da criança não haver sido retirada. Os dados de roupas e acessórios foram obtidos previamente em uma loja para crianças. Desta forma, quatro outros valores de massa corporal foram gerados pela medição realizada pelos profissionais $(\mathrm{V})$ e a lida pelo observador $\left(\mathrm{V}_{1}\right)$ :

$\mathrm{V}_{2}=$ obtida pelo profissional e corrigida pela calibração do equipamento;

$\mathrm{V}_{3}=$ lida pelo observador e corrigida pela não retirada da vestimenta;

$\mathrm{V}_{4}=$ lida pelo observador e corrigida pela calibração do equipamento;

$\mathrm{V}_{5}=$ lida pelo observador e corrigida pela não retirada da vestimenta e pela calibração do equipamento.

Os resultados das classificações nutricionais obtidas com os valores $\mathrm{V}_{1}, \mathrm{~V}_{2}, \mathrm{~V}_{3}, \mathrm{~V}_{4}$ e $\mathrm{V}_{5}$ foram relacionados com o $\mathrm{V}$, usando-se um quadro de contingência $2 \times 2$ para observar possíveis diferenças na classificação do estado nutricional das crianças. Além disso, o teste de tStudent pareado foi utilizado para comparar os valores de massa corporal produzidos pelo profissional (V) com as outras medições $\left(V_{1} a V_{5}\right)$.

Quanto à exatidão dos equipamentos, foram calculados coeficientes de correlação de Pearson (r) para medir o grau de correlação entre os valores de massas-padrão (x) e a média dos valores obtidos durante a calibração dos equipamentos (y). Foram calculadas equações de regressão linear com os valores encontrados 
na calibração para cada balança (Fletcher et al., 1988). Com essas equações, os valores de massa corporal obtidos pelo profissional foram corrigidos pelo equipamento, utilizando-se a fórmula $\mathrm{y}=\mathrm{ax}+\mathrm{b}$.

O coeficiente de Kappa de Cohen $(k)$ foi aplicado para se conhecer o nível de concordância entre as classificações nutricionais usando a medida produzida pelo profissional (V) contra as outras medidas $\left(V_{1}\right.$ a $\left.V_{5}\right)$, utilizando-se a fórmula: $k=\mathrm{Co}-\mathrm{Ca} / 1$ - Ca (onde Co = concordância observada e Ca = concordância devido ao acaso). A classificação de concordância por Kappa de Cohen foi a de Landis \& Koch (1977): leve $(0,00-0,20)$; razoável $(0,21-0,40)$; moderada $(0,41-0,60)$; substancial $(0,61-0,80)$; quase perfeita $(0,81-1,00)$.

\section{Resultados e discussão}

Do total de crianças, $46,9 \%(n=137)$ eram do sexo feminino e $53,1 \%(n=155)$ do sexo masculino. Mais da metade das crianças $(64,7 \%$; $\mathrm{n}=$ 189) foram pesadas em balança pediátrica mecânica; $29,8 \%$ ( $n=87$ ), na balança de plataforma mecânica e 5,5\% ( $n=16)$ na balança pediátrica eletrônica. Das crianças maiores de dois anos ( $\mathrm{n}=91), 16,5 \%$ foram medidas em balança pediátrica (mecânica ou eletrônica) e, das menores de dois anos $(n=201), 6,0 \%$ foram medidas em balança de plataforma mecânica.

Do total de CMS estudados, 11 tiveram a relação número de pediatras/número de auxiliares igual a um; cinco menor do que um e cinco maior do que um. Foram visitados dez turnos no período da tarde e 11 turnos no da manhã, distribuídos homogeneamente pelos dias da semana. Pôde-se observar que, dos 21 turnos, sete tiveram menos de dez crianças menores de cinco anos pesadas pelos profissionais de saúde. Verificou-se que, das vinte balanças pediátricas mecânicas presentes na sala de preparo, cinco estavam em posição incorreta (ao lado da janela ou porta de fluxo de pacientes) e 15 estavam apoiadas em local inadequado, encontrando-se, na sua grande maioria, apoiadas em mesas de ferro, pequenas, móveis e apresentando pontos de ferrujem, comprometendo, particularmente, seu nivelamento. A recomendação do INMETRO é que, para toda balança, principalmente a pediátrica eletrônica, deve-se ter um apoio fixo, revestido de aço inoxidável, para evitar erros na tara do equipamento. A única balança eletrônica avaliada estava posicionada e apoiada de forma correta. Das vinte balanças de plataforma, observou-se que 19 encontravam-se encostadas na parede, prejudicando a leitura do valor da medida de massa corporal na escala da balança e três estavam apoiadas em local inadequado (chão de cimento ou desnivelado).

Das 21 salas de preparo visitadas, 16 possuíam ambiente favorável para o trabalho do profissional de saúde. Neste caso, a única questão relatada pelos auxiliares destes CMS foi a de que o número de crianças para o atendimento havia aumentado, dificultando suas atividades de trabalho. Embora esta questão tivesse sido mencionada, observou-se que somente uma unidade teve 24 crianças pesadas por um auxiliar para o atendimento pediátrico, não interferindo em grande proporção na rotina do profissional de saúde, já que na rotina dos serviços o profissional de saúde atende vinte pacientes/dia. Nestas unidades, os profissionais de saúde começam a preparar a criança para o atendimento uma hora antes do início das consultas para evitar possíveis transtornos ou atrasos no período de medição. Observou-se que, no início da manhã (de $7 \mathrm{~h}$ às $8 \mathrm{~h}$ ), o número de crianças é grande e o trabalho do profissional de saúde é intenso. Entretanto, a partir das $9 \mathrm{~h}$, o profissional de saúde passa a diminuir suas atividades, parando às vezes por dez minutos para um café. A mesma rotina foi observada para o turno da tarde. O SISVAN estava implantado em 15 dos CMS estudados. Dois dos serviços que não possuíam esse sistema tiveram seus profissionais de saúde treinados quanto à técnica de pesagem num período inferior a três meses.

Dos CMS que possuíam SISVAN ( $\mathrm{n}=15)$, dez tinham profissionais de saúde treinados quanto à técnica de pesagem há mais de 24 meses; 4 acima de 3 meses e 1 com mais 12 meses. Apesar de muitas unidades apresentarem SISVAN implantado e a grande maioria dos profissionais de saúde ter sido capacitada quanto à técnica de pesagem, verificou-se que havia a necessidade de ser realizado um novo treinamento para reciclá-los, pois excedia em mais de 24 meses o período do último treinamento. Segundo Bittencourt et al. (1997), devido às peculiaridades da rotina que englobam os procedimentos de pesagem, sugere-se que estes devam ser avaliados, periodicamente, dentro do serviço. Isto porque é importante observar quando o profissional de saúde, quer por descuido, cansaço ou pela desvalorização do seu trabalho por outros profissionais, começa a perder o entusiasmo despertado no treinamento. Assim, sugere-se que haja uma reciclagem anual.

Dos procedimentos realizados pelos profissionais de saúde (Tabela 1), observou-se que aqueles de "nivelar o fiel da balança" e "esperar 
o braço da balança ficar alinhado com o fiel” não foram executados, respectivamente, em $21,9 \%$ e $44,2 \%$ do total das medições; em $26,8 \%$ e $46,8 \%$ daquelas feitas em balanças pediátricas e em $10,3 \%$ e $37,9 \%$ daquelas em balanças de plataforma. Observou-se ainda que, quando realizados, a maioria destes procedimentos foi feita de forma incorreta pelos profissionais de saúde (Tabela 2).
O procedimento de "despir a criança" foi realizado somente em $29,9 \%$ das observações feitas nas balanças de plataforma e, mesmo assim, 42,3\% destes não foram feitos de forma correta pelos profissionais de saúde (Tabela 2). Neste procedimento foi verificado que muitos profissionais só o realizavam para crianças menores de um ano. Na maioria das vezes, os profissionais diziam que não era necessário despir

Tabela 1

Freqüências percentuais da realização dos procedimentos da técnica de pesagem realizados em crianças menores de cinco anos atendidas nos Centros Municipais de Saúde por tipo de equipamento. Município do Rio de Janeiro, Julho/Setembro, 1996.

\begin{tabular}{|c|c|c|c|}
\hline Procedimentos & $\begin{array}{c}\text { Total } \\
(n=292)\end{array}$ & $\begin{array}{c}\text { Balança } \\
\text { pediátrica } \\
(n=205)\end{array}$ & $\begin{array}{c}\text { Balança de } \\
\text { plataforma } \\
\quad(n=87)\end{array}$ \\
\hline Nivelou o fiel da balança & 21,9 & 26,8 & 10,3 \\
\hline Despiu a criança & 75,7 & 95,1 & 29,9 \\
\hline Colocou a criança no centro da balança & 99,0 & 99,5 & 97,7 \\
\hline Esperou a criança ficar tranqüila & 89,4 & 89,8 & 88,5 \\
\hline Moveu o cursor dos quilos & $100,0 *$ & $100,0 * \star$ & 100,0 \\
\hline Moveu o cursor dos gramas & $100,0 *$ & $100,0 * \star$ & 100,0 \\
\hline $\begin{array}{l}\text { Esperou a agulha do braço da balança } \\
\text { ficar alinhada com o fiel }\end{array}$ & 44,2 & 46,8 & 37,9 \\
\hline $\begin{array}{l}\text { Leu o valor de massa corporal de frente } \\
\text { aos marcadores }\end{array}$ & 99,7 & 99,5 & 100,0 \\
\hline Registrou o valor de massa corporal & 100,0 & 100,0 & 100,0 \\
\hline
\end{tabular}

${ }^{\star} \mathrm{n}=276 ;{ }^{* \star} \mathrm{n}=189$

Tabela 2

Freqüências percentuais e absolutas dos procedimentos da técnica de pesagem realizados e de forma correta em crianças menores de cinco anos atendidas nos Centros Municipais de Saúde por tipo de equipamento. Município do Rio de Janeiro, Julho/Setembro, 1996.

\begin{tabular}{|c|c|c|c|c|c|c|}
\hline \multirow[t]{2}{*}{ Procedimentos } & \multicolumn{2}{|c|}{ Total } & \multicolumn{2}{|c|}{$\begin{array}{c}\text { Balança } \\
\text { pediátrica }\end{array}$} & \multicolumn{2}{|c|}{$\begin{array}{l}\text { Balança de } \\
\text { plataforma }\end{array}$} \\
\hline & $n^{*}$ & 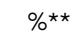 & $n$ & $\%$ & $n$ & $\%$ \\
\hline Nivelou o fiel da balança & 64 & 56,3 & 55 & 58,2 & 9 & 44,4 \\
\hline Despiu a criança & 221 & 71,5 & 194 & 75,4 & 26 & 42,3 \\
\hline Colocou a criança no centro da balança & 289 & 97,6 & 204 & 100,0 & 85 & 91,8 \\
\hline Esperou a criança ficar tranqüila & 261 & 95,8 & 184 & 94,6 & 77 & 98,7 \\
\hline Moveu o cursor dos quilos & 276 & 33,3 & 189 & 41,8 & 87 & 26,4 \\
\hline Moveu o cursor dos gramas & 276 & 33,3 & 189 & 41,8 & 87 & 28,7 \\
\hline $\begin{array}{l}\text { Esperou a agulha do braço da balança } \\
\text { ficar alinhada com o fiel }\end{array}$ & 129 & 57,2 & 96 & 56,3 & 33 & 36,4 \\
\hline Registrou o valor de massa corporal & 292 & 54,5 & 205 & 56,1 & 87 & 50,6 \\
\hline
\end{tabular}

* Números absolutos dos procedimentos realizados.

** Percentual de procedimentos realizados corretamente. 
a criança. Em um dos CMS visitados, a sala de medição não tinha porta, fazendo com que o profissional não realizasse o procedimento de despir as crianças para evitar que as mesmas ficassem expostas. Mesmo que não se tenha dito os objetivos do estudo aos profissionais de saúde da sala de medição, a fim de serem evitados viéses devido à presença de um observador na sala, percebeu-se que a sua presença muitas vezes levava o profissional de saúde a despir totalmente crianças menores de dois anos.

Os demais procedimentos foram realizados em mais de 3/4 das observações; todavia, quando foram observados quanto à sua realização ou não de forma correta, percebeu-se que, especialmente os procedimentos de "mover os cursores dos quilos" e "mover os cursores dos gramas" não foram realizados de forma correta em 33,3\% do total das observações; em 41,8\% das observações em balança pediátrica e em $26,4 \%$ e $28,7 \%$, respectivamente, das observações em balanças de plataforma (Tabela 2). Quando o profissional de saúde terminava de realizar a pesagem da criança, às vezes não retornava com os cursores para o zero da escala da balança. Desta forma, quando iniciava a pesagem seguinte, os cursores ainda estavam posicionados no valor obtido na pesagem anterior. Este procedimento implicava a não-realização da primeira etapa, que é a de "nivelar o fiel da balança”.

O desempenho dos profissionais treinados e não treinados quanto à técnica de pesagem foi semelhante, tanto em relação à realização dos procedimentos, quanto à realização de forma correta dos mesmos. Os dados demonstraram que, apesar de a grande maioria dos CMS possuir SISVAN implantado e, conseqüentemente, dispor de profissionais treinados quan- to às técnicas de pesagem, houve falhas em alguns procedimentos importantes para assegurar a qualidade do valor da medida de massa corporal. Verifica-se, porém, que o período de treinamento destes profissionais havia ocorrido, em sua maioria, há 24 meses ou mais antes do estudo, não tendo sido reciclados posteriormente. Observou-se, também, que alguns profissionais lotados em outros setores, às vezes, cobriam a falta daqueles da sala de preparo da pediatria e todos eles comentaram que não haviam recebido treinamento no período de implantação do SISVAN. Somente na graduação ou curso técnico haviam tido instruções quanto às técnicas de pesagem.

Na balança pediátrica eletrônica, todos os procedimentos foram realizados e de forma correta. Este resultado sugere maior facilidade e praticidade em se utilizar o equipamento. $\mathrm{Ob}$ servou-se, também, que a unidade apresentava apoio e posição adequados para o equipamento, favorecendo o trabalho do profissional de saúde.

Na calibração dos equipamentos, foram encontrados erros em todas as balanças pediátricas e em 12 das 20 balanças de plataforma. Contudo, os valores da calibração apresentaram uma correlação quase perfeita $(r=0,999)$. Quanto ao estado de conservação, 50\% das balanças apresentavam problemas.

Analisando a concordância entre os valores de massa corporal, observou-se que, do total das observações analisadas $(n=250), 25,5 \%$ das diferenças encontradas entre os valores de massa corporal produzidos pelo profissional e os lidos pelo observador não estavam dentro do intervalo de adequação proposto, sendo esta diferença estatisticamente significativa $(\mathrm{p}<$ 0,003 ), mas o valor médio foi de $23 \mathrm{~g}$ (Tabela 3 ).

Tabela 3

Médias, desvios-padrão (DP) e valores mínimos, máximos, t-Student e a probabilidade (p) das diferenças entre os valores absolutos de massa corporal ( $\mathrm{kg}$ ) produzidos pelos profissionais de saúde (V) e os lidos pelo observador, após várias correções, de crianças menores de cinco anos atendidas nos 21 Centros Municipais de Saúde. Município do Rio de Janeiro, Julho/Setembro, 1996.

\begin{tabular}{lccccccc}
\hline Diferença* & $\mathbf{n}$ & Média & DP & Mínimo & Máximo & t-Student & $\mathbf{p}$ \\
\hline V-V1 & 250 & $-0,023$ & 0,122 & $-0,700$ & 0,530 & $-3,022$ & 0,003 \\
V-V2 & 283 & 0,019 & 0,078 & $-0,170$ & 0,370 & 4,050 & 0,001 \\
V-V3 & 126 & 0,129 & 0,193 & $-0,300$ & 0,900 & 7,506 & 0,001 \\
V-V4 & 241 & $-0,009$ & 0,138 & $-0,750$ & 0,590 & $-1,024$ & 0,307 \\
V-V5 & 241 & 0,067 & 0,205 & $-0,750$ & 1,110 & 5,029 & 0,001 \\
\hline
\end{tabular}

* A definição das variáveis se encontra no texto. 
Do ponto de vista biológico, esta diferença não compromete expressivamente o valor da medida de massa corporal de um indivíduo, mesmo sendo este o de uma criança menor de dois anos, visto que este valor representa somente $0,21 \pm 1,66 \%$ de diferença. No entanto, os valores das diferenças variaram de $-700 \mathrm{~g}$ a $530 \mathrm{~g} \mathrm{em}$ $46,4 \%$ das vezes e nos outros a diferença foi zero. Assim sendo, no nível individual, crianças que tivessem com seus valores de massa corporal sub ou superestimados poderiam ter a classificação nutricional comprometida, notadamente as que tivessem suas medidas verdadeiras nos limites dos intervalos de confiança da normalidade. Interessante e inexplicavelmente, a única diferença média que não foi significativa (média de 9g, penúltima linha da Tabela 3) foi a existente entre o valor produzido pelo profissional (V) e o obtido pelo observador corrigido pela calibração do equipamento $\left(\mathrm{V}_{5}\right)$.

Na relação entre as classificações nutricionais realizadas com os valores de massa corporal produzidos pelos profissionais (V) e lidos pelo observador $\left(\mathrm{V}_{1}\right)$, o coeficiente de Kappa de Cohen indicou uma concordância perfeita $(\mathrm{k}=1)$ entre essas classificações (Tabela 4). Em geral, observou-se concordância entre todas as classificações nutricionais de $\mathrm{V}, \mathrm{V}_{1}, \mathrm{~V}_{3}, \mathrm{~V}_{4}$ e $\mathrm{V}_{5}$, quando relacionadas entre si, não ocorrendo o mesmo na relação entre as classificações nutricionais de $\mathrm{V} \operatorname{com} \mathrm{V}_{2}$, em que uma criança de "peso normal" foi classificada com "sobrepeso". Este achado pode ser explicado pelo fato de a classificação utilizada abarcar, em cada situação, (baixo peso, adequação e sobrepeso) uma grande faixa de valores de massa corporal. Desta forma, ainda que haja variações importantes entre os valores de massa corporal, não obrigatoriamente serão observadas alterações na classificação nutricional das crianças estudadas. Entretanto, na realidade dos serviços de saúde, estas modificações são importantes pa-

\begin{tabular}{|c|c|c|c|c|}
\hline \multicolumn{5}{|c|}{$\begin{array}{l}\text { Relação entre as classificações nutricionais dos valores de massa corporal } \\
\text { produzidos pelos profissionais de saúde (V) e os lidos pelo observador (V1), } \\
\text { de crianças menores de } 5 \text { anos atendidas nos } 21 \text { Centros Municipais de Saúde. } \\
\text { Município do Rio de Janeiro, Julho/Setembro, } 1996 .\end{array}$} \\
\hline \multirow[t]{2}{*}{ V } & \multicolumn{4}{|c|}{ V1 } \\
\hline & Baixo Peso & Adequação & Sobrepeso & Total \\
\hline Baixo Peso & $15(6 \%)$ & - & - & 15 \\
\hline Adequação & - & $222(88,8 \%)$ & - & 222 \\
\hline Sobrepeso & - & - & $13(5,2 \%)$ & 13 \\
\hline Total & 15 & 222 & 13 & 250 (100\%) \\
\hline
\end{tabular}

$K=1$

ra o diagnóstico e acompanhamento da evolução da criança, bem como para a conduta do profissional.

\section{Conclusão}

Apesar de os valores de massa corporal produzidos pelos profissionais de saúde não terem comprometido as classificações nutricionais das crianças avaliadas neste estudo, algumas variáveis, entre elas, a técnica de pesagem e o estado geral de alguns equipamentos, não tiveram resultados satisfatórios. Assim sendo, a capacitação bem como o treinamento periódico do profissional de saúde, a manutenção e a calibração dos equipamentos de pesagem devem ser realizadas periodicamente. Uma providência poderia ser a substituição do equipamento mecânico pelo eletrônico, devido à sua praticidade, garantindo, assim, valores fidedignos de massa corporal, tão importantes na prescrição médica e no acompanhamento nutricional da criança.

\section{Referências}

BATISTA FILHO, M., 1994. A Circunferência Braquial e o Índice entre os Perímetros Braquial e Cefálico como Métodos de Avaliação do Estado Nutricional de Crianças. Recife: Departamento de Nutrição, Centro de Ciências da Saúde, Universidade de Pernambuco. (mimeo.)

BITTENCOURT, S. A.; BARROS, D. C. \& MONTEIRO, K. A., 1997. Pesando e Medindo em uma Unidade de Saúde. Rio de Janeiro: Centro de Referência de Alimentação e Nutrição - Região Sudeste/Centro de Saúde Escola Germano Sinval Faria, Escola Nacional de Saúde Pública, Fundação Oswaldo Cruz. 
CHAVES, N., 1978. Requerimentos energéticos e protéicos: Discussão e estudo crítico. In: Nutrição Básica e Aplicada (N. Chaves, org.), pp. 85-93, Rio de Janeiro: Editora Guanabara Koogan.

DORNBRAND, L.; HOOLE, A. J. \& FLETCHER, R. H., 1990. Manual de Medicina Ambulatorial. Rio de Janeiro: MEDSI/Editora Médica e Científica.

FLETCHER, R. H.; FLETCHER, S. W. \&WAGNER, E. H., 1988. Clinical Epidemiology: The Essentials. 2nd Ed. Baltimore: Williams \& Wilkins.

HABICHT, J. P., 1974. Estandardización de métodos epidemiológicos cuantitativos sobre el terreno. Boletín de la Oficina Sanitária Panamericana, 76:375-384.

IEL (Instituto Euvaldo Lodi), 1994. Sistema Internacional de Unidades. v. 8. Rio de Janeiro: IEL.

INAN (Instituto Nacional de Alimentação e Nutrição)/MS (Ministério da Saúde), s.d. Padronização de Indicadores Antropométricos do Estado Nutricional: Elenco Mínimo. Brasília: INAN/MS.

INAN (Instituto Nacional de Alimentação e Nutrição)/MS (Ministério da Saúde), 1996. Antropometria: Manual de Técnicas e Procedimentos. Centro de Referência em Vigilância Nutricional da Região Centro-Oeste. Goiânia: Universidade Federal de Goiás.

INMETRO (Instituto Nacional de Metrologia, Normalização e Qualidade Industrial), 1995. Vocabulário Internacional de Termos Fundamentais e Gerais de Metrologia. Duque de Caxias: INMETRO.

LANDIS, J. R. \& KOCH, G., 1977. The measurement of observer agreement for categorical data. Biometrics, 33:159-174.

MASON, J. B.; HABICHT, J.; TABATABAI, H. \& VALVERDE, V., 1984. Vigilância Nutricional. Genebra: Organização Mundial de Saúde.
MONTEIRO, C. A.; BENÍCIO, M. H. D. \& IUNES, R., 1992. O estado nutricional de crianças brasileiras: A trajetória de 1975 a 1989. In: Aspectos de Saúde e Nutrição de Crianças no Brasil. Perfil Estatístico de Crianças e Mães no Brasil (F. G. Monteiro \& R. Cervini, org.), pp. 43-59, Rio de Janeiro: Fundo das Nações Unidas para a Infância/Instituto Nacional de Alimentação e Nutrição/Fundação Instituto Brasileiro de Geografia e Estatística.

MUELLER, W. H. \& MARTOREL, R., 1988. Reability and accuracy of measurement. In: Anthropometric Standardization Reference Manual (T. G. Lohmann, A. F. Roche \& R. Martorell, ed.), pp. 8386, Champaign: Human Kinetics Books.

NACIONES UNIDAS, 1988. Como Pesar y Medir Niños: Determinación del Estado Nutricional de Niños Pequeños Mediante Encuestas de Hogares. New York: Departamento de Cooperación Técnica para el Desarrollo y Oficina de Estadística, Naciones Unidas/Fondo de las Naciones Unidas para la Infancia.

NCHS (National Center for Health Statistics), 1977. NCHS Growth Curves for Children Birth - 18 Years United States. Vital and Health Statistics Series 11 no 165. DHEW Publication, pp. 78-165, Washington, DC: U. S. Printing Office.

WHO (World Health Organization), 1995. Physical Status: The Use and Interpretation of Physical Status: The Use and Interpretation of Anthropometry. WHO Technical Report Series 854. Geneva: WHO.

Recebido em 3 de julho de 2000

Versão final reapresentada em 3 de abril de 2001

Aprovado em 7 de junho de 2001 